\title{
A Pillar-Shaped Antifuse-Based Silicon Chemical Sensor and Actuator
}

\author{
Alexey Y. Kovalgin, Jisk Holleman, and Gheorghe (Gigi) Iordache
}

\begin{abstract}
We designed a silicon-processing compatible, simple, and cheap device operating at a power down to sub- $\mu \mathrm{W}$. It has a pillar-shaped structure with a nanoscopic $(10-100 \mathrm{~nm}$ in size) conductive link (the so-called antifuse) created between two electrodes separated by a $\mathrm{SiO}_{2}$ layer. The device exhibits a diode-like behavior due to the depletion effects in the mono-silicon pillar. The device is capable of maintaining a microscopic hot-surface area of several hundreds degrees centigrade. The size of the hot area and its temperature can be manipulated by the sign of the applied bias. Two different heat-generation mechanisms (i.e., dissipation at a resistor and a non-radiative recombination of carriers) are proposed and modelled. Such a device can be used as a heat source, as a light source, and as a sensitive detector of light and heat. In this paper, we describe thermo-electrical properties of the fabricated devices and demonstrate their feasibility to perform as gas-, adsorption-, desorption sensors, and as units for activating chemisorption/decomposition of gaseous precursors, i.e., micro-reactors.
\end{abstract}

Index Terms-Antifuse, chemical actuator, chemical sensor, micro-reactor.

\section{INTRODUCTION}

A MONG a large variety of chemical sensors and chemical actuators being developed, electrical devices utilizing hot surfaces, e.g., employing hot filaments or hot plates, are of great importance. Hot-surface devices are often used in chemical sensors [1], [2] or gas mass-flow meters based on temperature changes. Such devices require elevated temperatures to operate in the range between 100 and $600^{\circ} \mathrm{C}$. Because of the elevated operating temperatures, the devices (optionally coated with catalytically active surface coatings) can also behave as chemical actuators, i.e., micro-reactors providing energy in the form of heat to initiate thermo-activated chemical reactions.

One significant drawback of conventional devices utilizing hot surfaces is their relatively high power consumption. Apart from enabling portable, battery operated systems, the low power needed to generate high temperatures in a short time is of special interest, e.g., for the study of dynamic transient chemical and physical processes. The commercial devices use, for example,

Manuscript received March 16, 2006; revised June 2, 2006; accepted June 2, 2006. This work was supported by EU under Project GRD1-1999-10849. This is an expanded paper from the Sensors 2005 Conference. The associate editor coordinating the review of this paper and approving it for publication was Prof. Ignacio Matias.

The authors are with the MESA+ Institute for Nanotechnology, University of Twente, 7500 AE Enschede, The Netherlands (e-mail: A.Y.Kovalgin@utwente.nl; J.Holleman@utwente.nl; giordache@go.com).

Color versions of one or more of the figures in this paper are available online at http://ieeexplore.ieee.org.

Digital Object Identifier 10.1109/JSEN.2006.888602 a platinum wire as a heating element and require a power of about $100 \mathrm{~mW}$. Other types of hot-plates that have been realized more recently on suspended membranes, employ a meander-like pattern of a conductive layer as platinum or poly-silicon and still require about $20-40 \mathrm{~mW}$ [3].

Looking forward to future development of the equipment intended for characterization and chemical actuation of microscopic and further nanoscopic quantities of matter, there is a great demand for low-power, simple, and cheap devices. A variety of such sensing/actuating devices can be embedded into a multifunctional lab-on-a-chip system and there actuate and in situ characterize physical and chemical processes on a micro- and nanometer scale. These practical challenges will open a route to a number of scientific challenges such as, e.g., in situ monitoring of reaction occurrence and reaction products. As an example, one can think of studying the dynamics of transient adsorption-desorption, chemisorption, or combustion processes, e.g., by measuring the reaction heat. Further, this can allow determining the local chemical kinetics. A CMOS-compatible technology is desired to enable the low-cost production in large quantities and in large arrays in standard IC factories with a minimum of dedicated post processing and a short development period.

In this paper, we present a new, simple, and cheap device (the so-called pillar-shaped antifuse), based on the idea of maintaining a microscopic hot surface area employing a nanoscopic conductive link created between two silicon electrodes separated by a dielectric [4], [5]. For such a pillar-shaped structure, the heat is mainly generated due to a non-radiative recombination of carriers in a thin poly-Si surface layer [6]. The result is that an extended poly-Si surface area instantly becomes hot and the vertical heat losses are decreased. Finally, this leads to an increased efficiency of the device. It appears from our measurements and modeling that whereas the entire surface electrode behaves as a heat generator, the device itself can perform as a sensitive heat detector [7], [8]. These properties combined with extremely low operating power down to a (sub)- $\mu \mathrm{W}$ range can give rise to a number of practical applications, e.g., in the field of chemical sensors, mass flow sensors, and temperature detectors. When integrated on a chip, such devices can allow in situ characterization and activation of physical and chemical processes on micro/nano scale and therefore contribute to some of the present-day scientific challenges (e.g., molecular electronics). We developed silicon-processing compatible, simple and low cost methods for fabrication of the devices. This makes it more compatible with standard CMOS processing. 


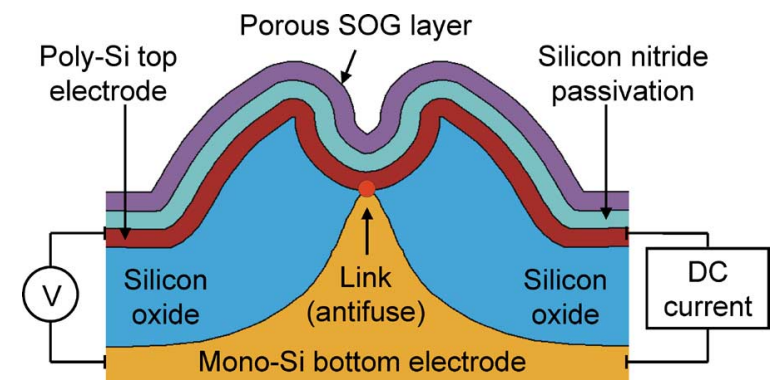

Fig. 1. Schematic cross-sectional view of a PSA-device.

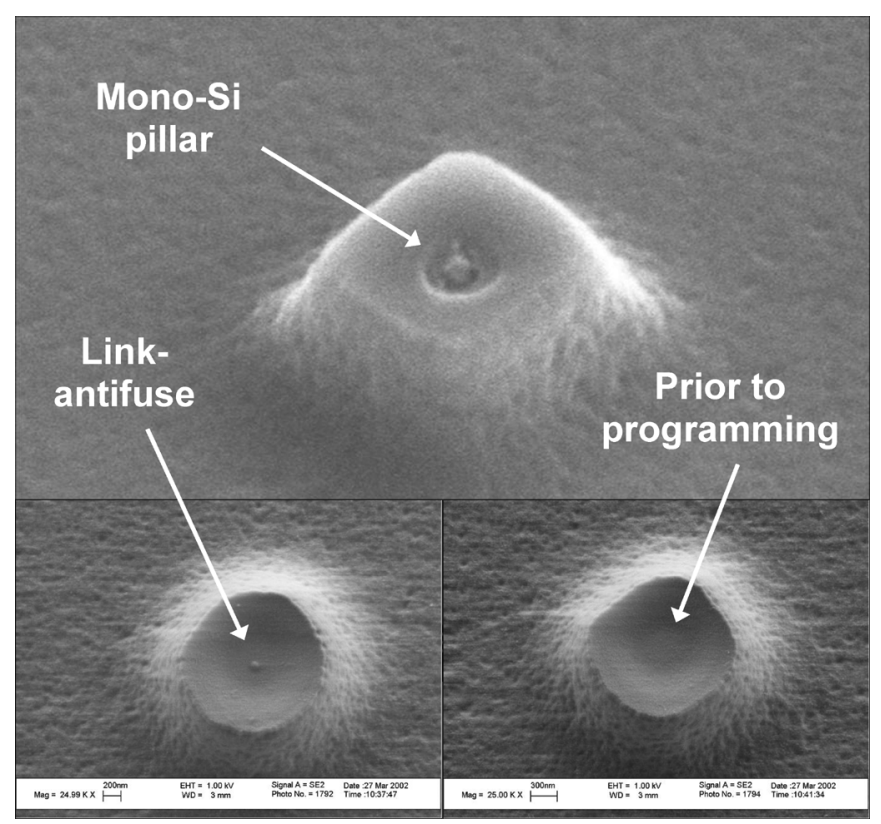

Fig. 2. SEM top-view images of various PSA-devices. (a) Before programming, lower-right corner. (b) After programming at $1 \mathrm{~mW}$, a link-antifuse is formed in the center, lower-left corner. (c) Cavity is etched in the center enabling seeing the bottom mono-Si electrode (upper image).

\section{DEVICE DESIGN AND REALIZATION}

\section{A. Design}

Our device resembles a micron-sized volcano (see Figs. 1 and 2) with a nanoscopic conductive link formed in the center by means of controllable electrical programming. The structure consists of two electrodes: rather thick lowly-doped n-type mono-Si pillar-shaped bottom electrode and a very thin $(13 \mathrm{~nm})$ highly-doped n-type poly-Si top electrode, passivated with a 10-nm thick silicon nitride layer on top. A 10-nm thick silicon dioxide layer is deposited in between the two electrodes to separate them and to avoid an electrical contact. The surrounding 400-nm thick LOCOS oxide is needed a) for thermo-electrical isolation and b) to confine the area of the link formation at the center. The structure has a cylindrical symmetry along the line perpendicular to the central point on the surface.

Prior to electrical programming, i.e., before a conductive link between the electrodes is established through the oxide, there is a very low leakage current flowing between the electrodes. To create the link, the insulating $\mathrm{SiO}_{2}$ layer between the electrodes is locally destroyed by a two-step electrical stress. During the first step, the insulator breakdown occurs due to a constant current stress, and the initial conductive link (path) appears. As it is generally accepted, the conductive breakdown part in oxides is caused by the creation of traps. During the second step called programming, a gradually increasing current is applied, adjusting the link resistance in a controllable manner. Such a conductive link is called antifuse [4], [5], meaning that insulator becomes conductive after fusing, contrary to a conventional fuse. Because of the pillar-shaped structure with the conductive link-antifuse in the center, the device is called a Pillar-Shaped Antifuse or a PSA-device.

Depending on programming current, the link-antifuse size varies between 10 and $100 \mathrm{~nm}$, as simulated and measured in [4] and [5]. A PSA-device requires a power in $\mu \mathrm{W}$-(sub)milliwatt range to keep a surface of one micron in diameter at a temperature of a few hundred degrees centigrade. The link resistance can finely be tuned in the range between hundreds of $\mathrm{G} \Omega$ and a few $\mathrm{k} \Omega$ by applying the appropriate current (power). This allows for tuning of the electrical resistance and, therefore, consumed electrical power for each device individually. It appears from our research that, as the link-antifuse is once created at a certain value of the programming current or programming power, its electrical properties cannot be changed under a stress below the programming value.

As further to be confirmed in Section IV, the PSA-devices exhibit two heat generation mechanisms. First, if a positive voltage is applied to the upper poly-electrode, the dissipation of heat occurs inside the link-antifuse acting as a resistor. If a negative bias is applied to the upper poly-electrode, the device can generate heat due to a non-radiative carrier recombination in this thin electrode. Furthermore, when negative bias is applied, a PSA-device additionally acts as a sensitive heat detector (i.e., thermo resistor).

Apart from the low power consumption, an advantage of the proposed device concept is decoupling of the electrical and thermal resistances. In a conventional hot-plate device, the heat is generated either by a filament heater or suspended meander. In such a design, an increase of the thermal resistance of the electrodes is very important to minimize the heat losses through the electrodes. Indeed, an increase of the thermal resistance can only be achieved by thinning the electrodes, which leads to an increase of the electrical resistance and results in undesired extra power dissipation in the electrodes. Our device allows for an independent control of both the resistances because the electrical resistance is mostly determined by the link resistance. Therefore, thinning the upper poly-Si electrode does not affect (to some extents) electrical resistance of the entire PSA-device.

\section{B. Realization}

The process flow employed standard LOCOS oxidation and CVD technology. As the first step, a LPCVD silicon nitride film was deposited on an oxidized phosphorus-doped (100)-oriented silicon wafer with a resistivity of $2-5 \Omega \cdot \mathrm{cm}$. After patterning the

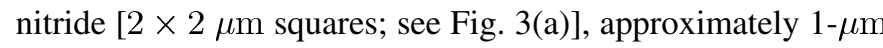
high silicon pillars, covered with the nitride caps, were formed on the wafer surface using isotropic $\mathrm{CF}_{4}-\mathrm{O}_{2}$ plasma etching [see Fig. 3(b)]. The following LOCOS oxidation at $1050^{\circ} \mathrm{C}$ provided sharpening the pillars and formation of a 400-nm silicon 
(a)

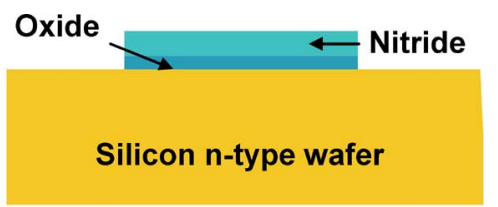

(b)

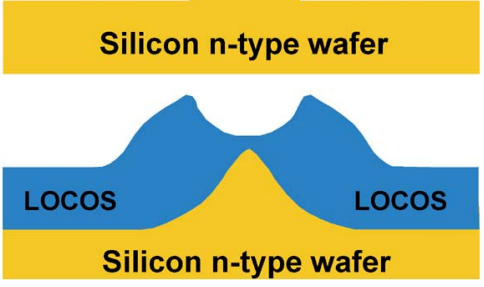

Fig. 3. Processing steps for making a PSA-device.

oxide for thermo-electrical isolation of the pillars. Further, the silicon nitride was etched away, and a thin gate oxide $(10 \mathrm{~nm})$ was grown on the silicon tip surface [see Fig. 3(c)]. Then, a 13-nm thick in situ phosphorus-doped silicon layer (doping $10^{20} \mathrm{at} / \mathrm{cm}^{-3}$ ) was deposited on the wafer surface, followed by LPCVD of a 10-nm thick silicon nitride layer for passivation of the device surface. Patterning the silicon $/ \mathrm{Si}_{3} \mathrm{~N}_{4}$ areas, annealing, opening the contact windows, sputtering, and patterning aluminum electrodes for contact metallization finalized the device.

One should bear in mind that the LOCOS oxidation step is rather crucial because a short overoxidation time leads to the appearance of cavities in mono-silicon instead of sharp pillars. The deposition of a thin silicon layer, in situ doped with phosphorus, is a second important step. Such a layer is expected to meet a number of important requirements. First, the layer has to be reasonably thin in order to a) minimize the lateral heat losses and b) increase the heat generation efficiency due to the carrier recombination. Second, the layer electrical resistivity is needed to be sufficiently low to conduct the required electric current. From our experiments on deposition of the phosphorous-doped silicon films, heavily doped layers with a minimum resistivity of $1.4 \mathrm{~m} \Omega \mathrm{cm}$ (for a 100-200-nm thick film) can be deposited at $600^{\circ} \mathrm{C}$ from a mixture with a $\mathrm{PH}_{3} / \mathrm{SiH}_{4}$ ratio of 0.0013 in the gas phase. However, we observed a tremendous increase of the resistivity for the films thinner than $20 \mathrm{~nm}$. The resistivity increased up to $300 \mathrm{~m} \Omega \mathrm{cm}$ for a 10-nm thick film due to the surface/interface influence and grain boundaries. Grain boundaries can significantly contribute to the device resistance due to a larger number of grains, which are inherently smaller in size. This larger number is caused by the nucleation process and can further be reduced for a thicker layer because of the evolutionary selection [9].

Using the spin-on-glass technology, a number of our PSA-devices were coated with a porous Spin-On-Glass (SOG) layer that was approximately $350 \mathrm{~nm}$ thick. This step was done to explore the device feasibility to monitor heat-enhanced adsorption and desorption processes in the porous layer. Briefly, the SOG-solution was prepared and applied to the substrate by spinning. During the next drying step, there was a reduction by more than
$50 \%$ in weight and volume of the material. Yet the film remained adherent and continuous and maintained complete surface coverage. It has been shown that up to one micron of thickness, all shrinkage was taken up in the dimension perpendicular to the surface and not in the plane of the substrate. Next, further heat treatment was applied to harden the layer. Although the micro porosity of silica is not removed entirely until $1000^{\circ} \mathrm{C}$, it may already behave as an oxidant barrier or passivation coating at $600^{\circ} \mathrm{C}$. As the processing temperatures we used are below $400^{\circ} \mathrm{C}$, the obtained SOG film is a rather porous material.

\section{Physical Properties}

During the electrical $I-V$ measurements discussed below, we normally applied a voltage between the electrodes (increased in small steps) and measured the current. Both the programming and electrical characterization were carried out using a parameter analyzer Agilent 4156C.

After the device fabrication, a conductive link-antifuse was initially created by applying a fixed current in the sub-nanoampere range to enable a breakdown of the $10-\mathrm{nm}$ thick $\mathrm{SiO}_{2}$ layer in between the electrodes. During this step, a negative (or positive) bias of several volts was applied to the poly-Si electrode. A series resistor of $1 \mathrm{M} \Omega$ was connected to the device to prevent an uncontrollable current increase during the breakdown.

Fig. 4 illustrates a change of the device resistance (mainly the link resistance) with sweeping the bias after the dielectric breakdown. For measurements under the conditions presented in this figure, there was no difference between the sign of the bias (i.e., positive or negative) applied to the poly-Si upper electrode. For the first time after the dielectric breakdown, the bias was swept up to $-5 \mathrm{~V}$. With the increase up to $-3 \mathrm{~V}$, we observed a gradual increase of the resistance with a maximum at $200 \mathrm{G} \Omega$. The further increase of bias up to $-5 \mathrm{~V}$ resulted in a gradual decrease of the resistance down to $67 \mathrm{G} \Omega$. For the second voltage sweep between 0 and $-7 \mathrm{~V}$, all the resistances showed lower values resulted in a lower curve. The third voltage sweep up to $-8 \mathrm{~V}$ resulted in even lower resistances. Continuing the sequence, one should obtain a further decrease of the resistance values. The latter is only correct when the maximum sweep voltage for every next step is higher compared to that for the previous step. At such low dissipated powers not exceeding $2 \mathrm{nW}$ and combined with a long sweep time in the range of minutes, one can hardly expect sufficient link heating. The overall resistance decrease can probably be attributed to the formation of new traps in the oxide between the electrodes.

At this stage of device programming, one can further switch to applying a positive bias only, until a power of several milliewatts is achieved. One can notice a continuous decrease of the device resistance down to several $\mathrm{k} \Omega$ with increasing power (see Fig. 5). As this power is already sufficient to influence the antifuse temperature, such a resistance decrease can occur due to heating the antifuse and poly-Si electrode surface. As described by Seto's model [10], the resistance of poly-Si is determined by the resistances of Si crystals and grain boundaries. With increasing temperature, more charge carriers are able to overcome the grain barriers, which results in a negative temperature coefficient for the resistance (TCR). On the other hand, the TCR of 


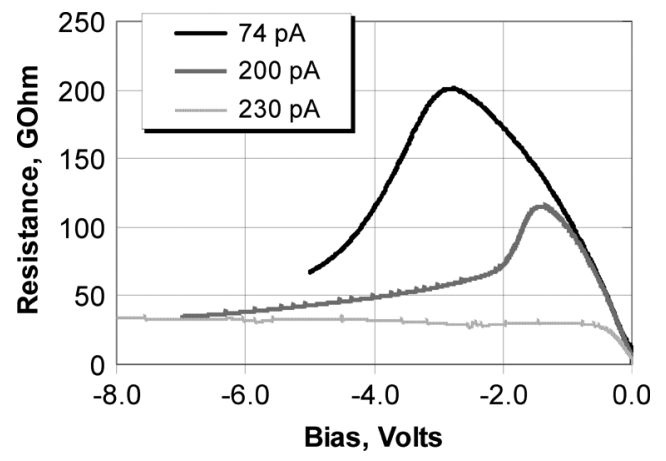

Fig. 4. Dependence of link-antifuse resistance on applied bias at first stages of device programming. Maximum programming current for each curve is printed in the legend.

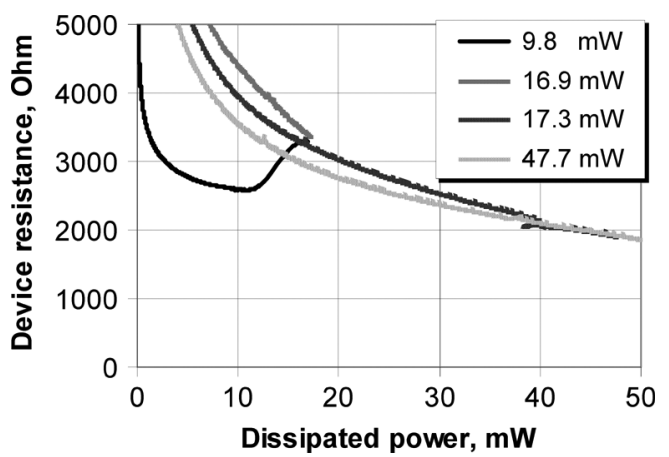

Fig. 5. Dependence of device resistance on applied power for a sequence of several power sweeps. A positive bias is applied to the poly-Si electrode. Maximum programming power preceding the acquisition of each curve is printed in the legend.

the sufficiently doped Si crystallites is positive due to the increased scattering effects. As doping enhances the influence of the crystallites, positive TCR values for highly doped poly-Si films are reported [11]. In our case, due to a large number of small grains expected for such a thin poly-Si upper electrode, we assume a dominant influence of the grain boundaries (confirmed by the dramatic resistivity increase for the films thinner than $20 \mathrm{~nm}$, see Section II) and, therefore, a negative TCR.

At a certain moment, the high antifuse temperature results in an irreversible resistance increases (see Fig. 5). This effect can be caused by an irreversible change in the material quality, occurring either in the link itself or in the upper poly-Si electrode. For example, diffusion of oxygen atoms to the link or evaporation of the impurities from the thin poly electrode can take place. It should be noticed that approaching this point causes sometimes a rather noisy resistance behavior. Finally, applying a power of $20-40 \mathrm{~mW}$ results in an irreversible resistance decrease, which can be caused by gradual enlarging the link due to its re-melting ( $1688 \mathrm{~K}$ for $\mathrm{Si}$ and $\sim 1900 \mathrm{~K}$ for $\mathrm{SiO}_{2}$ ).

It appears from our research that as the link-antifuse is once created at a certain value of the programming current (power), its electrical properties cannot be changed under a stress below the programming value. This means that once realized dependence of resistance on power can be multiply repeated, provided that the maximum programming current (power) is not exceeded. Exceeding the maximum level causes re-programming of the link.

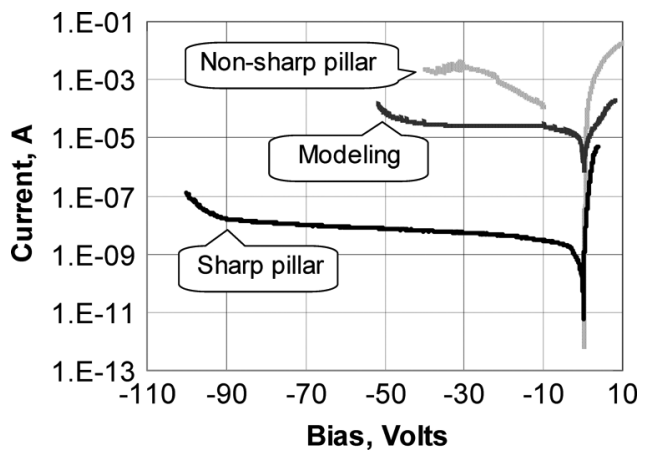

Fig. 6. Diode-like $I-V$ characteristics of PSA-devices (both electrodes are doped with phosphorous) with a different degree of sharpness of the mono-Si pillar.

When, instead of applying a positive bias, a negative bias sweep is applied to the poly-Si electrode after the dielectric breakdown, the PSA-devices exhibit much higher electrical resistance compared to positive biasing. In this light, a PSA-device with the same type of electrode doping behaves similar to a diode (see Fig. 6). This rather interesting result can be attributed to depletion-related effects in the mono-silicon electrode at a negative bias. These effects can lead to the formation of a depletion or even inversion layer (induced by the applied electric field) in the silicon pillar and require the existence of a leaky capacitor. Such a capacitor can only be formed for a) sufficiently small links preventing the creation of a short circuit between the electrodes and b) sufficiently sharp pillars enhancing the field effect. Fig. 7 illustrates the formation of an inversion layer and explains a qualitative difference between a "sharp" and "non-sharp" pillar.

The presence of a leaky capacitor was confirmed by the following observations. First, the measurements were carried out for a number of PSA-devices with a different degree of sharpness of the pillar. It appeared that only rather sharp pillars were able to maintain low currents under high negative biases up to $-100 \mathrm{~V}$. If radius of the tip increased (i.e., less sharp pillar; see Figs. 6 and 7), the diode-like behavior deteriorated and finally disappeared for certain radius. Second, the device ability to maintain low currents at high negative biases was also affected by the link size. A low current at a high negative bias could only be established for small links (i.e., very low programming currents), which were not able to suppress the depletion by injecting into the pillar a sufficient amount of carriers (i.e., electrons) from the heavily doped poly-Si electrode. Third, our simulations (to be presented in the next section) confirm the formation of an inversion layer in the silicon pillar and predict the measured diode-like behavior.

Resuming, the realized PSA-devices exhibit non-trivial $I-V$ characteristics (see Fig. 6). At near-zero bias, the device resistance is very high. A gradual increase of the bias (positive sign, applied to the poly-Si electrode) leads to a rather sharp increase of the current. Applying a negative bias causes much higher device resistance compared to positive biasing. Such a diode-like behavior can be attributed to depletion-related effects in the mono-Si electrode at a negative bias. 


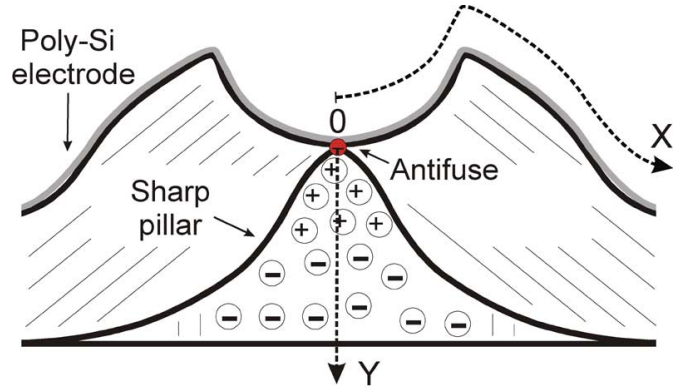

(a)

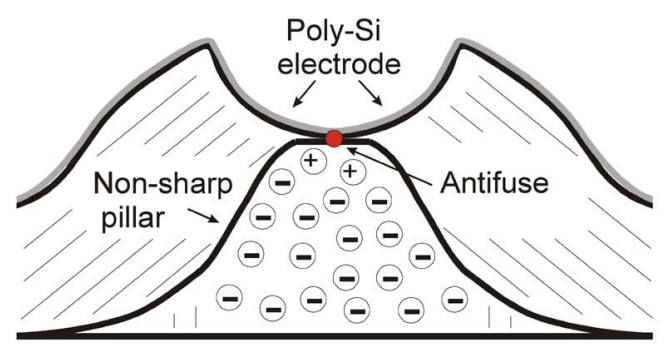

(b)

Fig. 7. Schematic formation of an inversion layer for a (a) sharp and (b) nonsharp PSA-device. Negative bias is applied to the poly-Si electrode. The modeled concentration of holes, extracted along vertical y-axis, is depicted in Fig. 9. The surface temperature, extracted from the cross-section made through the non-planar surface layer along x-axis, is shown in Fig. 10. The zero point corresponds to the antifuse position.

From this, one can expect two different mechanisms of heat generation, as further to be confirmed in Section IV. If a positive bias is applied to the upper poly-Si electrode, the device acts as a resistor. The heat is locally dissipated in the center and further distributed in lateral and vertical directions accordingly to thermal conductivities of the materials used. The lateral heat transport is limited due to the small thickness of the poly-layer. The vertical heat transport and, therefore, the heat losses downwards are enhanced because the massive silicon pillar is close to the heat dissipation center. In this regime, the efficiency of the heat generation is low due to the vertical losses. When a negative bias is applied to the upper poly-Si electrode, an inversion layer can be formed in the silicon pillar under certain conditions. This enhances the carrier recombination (i.e., recombination of holes injected from the inversion layer in the pillar and electrons from the poly-Si electrode), which occurs along an extended surface area $(\sim 2 \mu \mathrm{m})$ of the thin upper electrode [6]. If such a process occurs, the result is that the entire surface area instantly becomes hot. The vertical heat losses through the pillar decrease due to the expanded size of the heat generation area. Therefore, the efficiency of the heat generation will increase compared to the first mechanism. Furthermore, the probability of device reprogramming will decrease compared to the positively biased regime, as the link-antifuse does not act as a heat dissipation center.

As a second important feature of our PSA-devices, a light emission in visible and near-IR region can be mentioned (see Fig. 8). This can be utilized, e.g., in micro-reactors to enable photo-activated processes. The spectra show a clearly distinguishable emission peak at about $700-720 \mathrm{~nm}$. To verify a

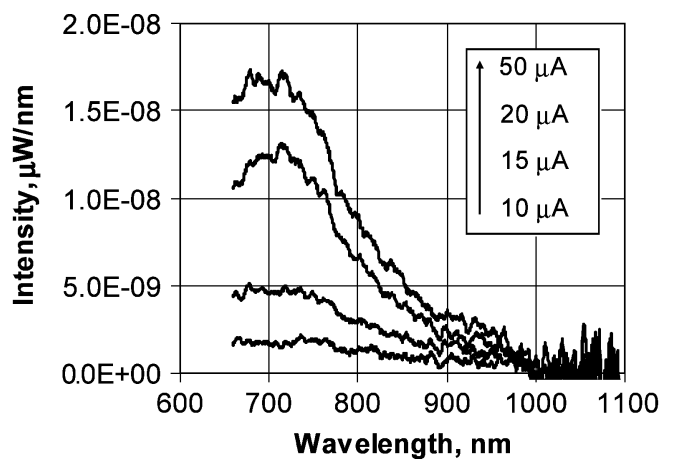

Fig. 8. Light emission spectra from a PSA-device plotted for various applied currents.

thermal origin of the peak, we simulated the emission sectra defined as a product of the calculated emissivity $\varepsilon(\lambda, T)$ and pure black body radiation.

The emissivity was calculated for the following stack of three thin layers: a 10 -nm thick $\mathrm{SiO}_{2}$, a 13-nm thick poly-Si electrode, and a 10-nm thick silicon nitride passivation layer on top. The data to calculate the optical properties of silicon at elevated temperatures were taken from [12]-[14]. The optical properties of the other materials used were assumed to be independent on temperature. For the modeled stack of layers, the calculated emissivity $\varepsilon(\lambda, T)$ decreased from $0.43-0.48$ to $0.29-0.34$ for the wavelength increased from $700 \mathrm{~nm}$ to $1000 \mathrm{~nm}$, in the temperature range $25-600^{\circ} \mathrm{C}$. The simulated spectra exhibited a gradual increase of the intensity with increasing the wavelength without showing any shoulder on it. From this, one can conclude that the emission spectra can not be attributed to the thermal emission and optical properties of the materials used. Most likely such a significant shift to higher energies $(720 \mathrm{~nm}$ corresponds to $1.72 \mathrm{eV}$ instead of the expected value of $1.1 \mathrm{eV}$ ) can be related to the recombination of hot carriers in the thin upper electrode or to the bandgap increase due to the small thickness of the electrode [15].

\section{NUMERICAL SIMULATIONS}

To confirm the two mechanisms of heat generation suggested in Section III, we carried out numerical simulations using ATLAS code (SILVACO International). Additionally, we aimed at verifying the ability of the PSA-devices to maintain a surface temperature of several hundreds degrees centigrade at low powers. ATLAS code was extended with GIGA module for lattice heat flow and general thermal environments. The module accounted for the dependence of material and transport parameters on the lattice temperature. The lattice temperature model included Joule heating, heating and cooling due to carrier generation and recombination, and the Peltier and Thomson effects. Free convection and radiative heat transfer were not included into the model.

As depicted in Fig. 1, we created a cylindrically-symmetrical structure for the simulations. We simulated the $I-V$ curves and temperature distributions by applying a voltage in small steps and calculating the current and temperature. For boundary conditions, two thermal contacts were specified. The contact (i.e., 


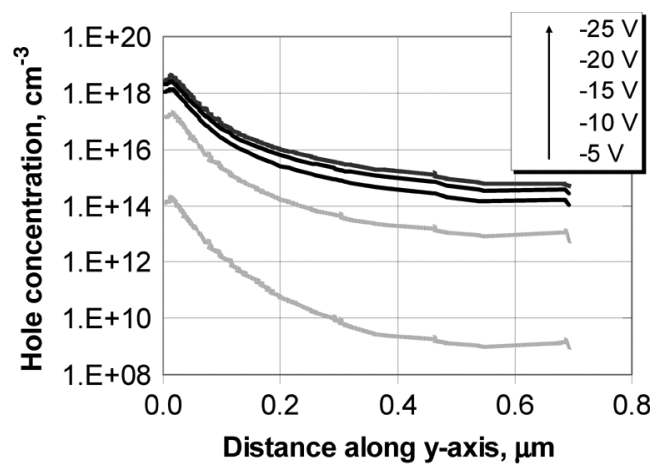

Fig. 9. Modeled concentration of holes along vertical y-axis (see Fig. 7) at several negative biases applied to the poly-Si electrode. The zero point corresponds to the antifuse position. The antifuse is $10 \mathrm{~nm}$ in diameter.

bulk silicon situated $30-50 \mu \mathrm{m}$ away from the antifuse) temperatures were set to $300 \mathrm{~K}$. The same held for the initial device/ambient temperatures.

To simulate (steady-state calculations only) the heat flow through the device and surrounding air, thermal conductivities of all the materials used were taken into account. The temperature-dependent thermal conductivity data for bulk silicon $\left(1.3 \mathrm{~W} \cdot \mathrm{cm}^{-1} \cdot \mathrm{K}^{-1}\right.$ at $\left.300 \mathrm{~K}\right)$ were extracted from the SILVACO database, verified, and accounted for their dependence on doping using data from [16]. The thermal conductivity of polycrystalline silicon $\left(0.15-0.5 \mathrm{~W} \cdot \mathrm{cm}^{-1} \cdot \mathrm{K}^{-1}\right.$ at $300 \mathrm{~K}$ ) was published to be lower than that of bulk silicon, depending on doping level [17] and layer thickness [18]. For thermally grown silicon oxide (thermal conductivity of $0.014 \mathrm{~W} \cdot \mathrm{cm}^{-1} \cdot \mathrm{K}^{-1}$ at $300 \mathrm{~K}$ ), the observed thermal conductivity decreased for thicknesses below approximately $250 \mathrm{~nm}$ due to the interface-substrate influence [19]. For thin silicon nitride films, the thermal conductivity was verified from [20] and assumed to be independent on temperature and thickness. The temperature-dependent thermal conductivity of dry air $\left(0.00025 \mathrm{~W} \cdot \mathrm{cm}^{-1} \cdot \mathrm{K}^{-1}\right.$ at $\left.300 \mathrm{~K}\right)$ was taken from [21] and compared with the values for humid air from [22].

The $I-V$ curve for a link of $10 \mathrm{~nm}$ in diameter was simulated in the voltage range between -50 and $10 \mathrm{~V}$ and resembled the experimental curves (see Fig. 6). To simulate for such high negative voltages, the lattice temperature module was turned off to eliminate convergence problems in the simulator. Instead, the model was accounted for field-dependent carrier mobility, depletion and inversion effects in silicon. A good qualitative agreement with the measurements confirmed that these effects could cause such a diode-like behavior. To confirm the presence of a leaky capacitor mentioned in Section III and formation of an inversion layer, we simulated the concentration of holes in the pillar at different applied voltages (see Fig. 9). The concentration was extracted along vertical y-axis, as depicted in Fig. 7. The zero point corresponded to the antifuse position. One can see that the concentration of holes significantly increased already at $-5 \mathrm{~V}$. The inversion layer started to form at a bias of $-15 \mathrm{~V}$. This behavior disappeared for the links bigger than a few tens of nanometers in diameter.

As mentioned, when a positive bias is applied to the poly-Si electrode, the heat is dissipated in the link-antifuse only and

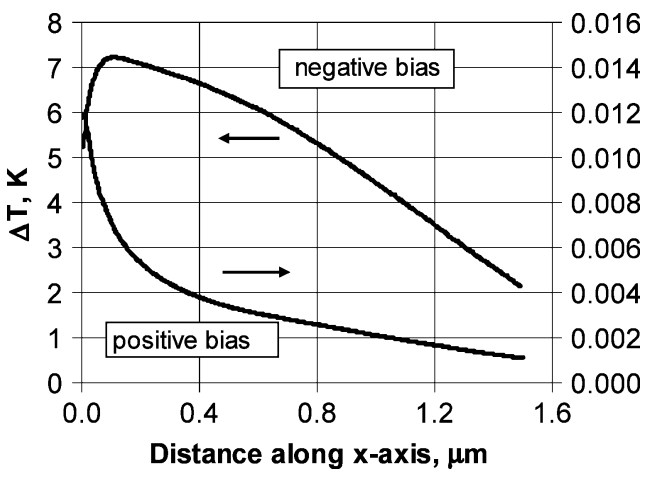

Fig. 10. Surface-temperature profiles simulated at the same value of dissipated power of $0.1 \mu \mathrm{W}$. An increase of the surface temperature $(\Delta T)$ above the thermal contact temperature is plotted versus distance from the link-antifuse. A comparison between applying a positive bias and applying a negative bias to the poly-Si electrode is shown. The temperature is extracted from the cross-section made through the non-planar surface layer along $\mathrm{x}$-axis (see Fig. 7). The zero point corresponds to the antifuse position. The antifuse is $20 \mathrm{~nm}$ in diameter.

is further distributed in lateral and vertical directions. The surface-temperature distribution shown in Fig. 10 indicates that the heat is dissipated in the center of the structure, i.e., at the link-antifuse. The temperature is extracted from the cross-section made through the non-planar surface layer along X-axis (see Fig. 7). The zero point corresponds to the antifuse position. When a negative bias is applied to the poly-Si electrode, the inversion layer is formed in the pillar and the heat generation mechanism based on a non-radiative carrier recombination is activated. Indeed, it is possible to see from the simulations that, in this regime, the carrier recombination intensively occurs along the surface area of the poly-electrode. This area is much larger with respect to the nanoscopic link area. As the entire area instantly becomes hot, the lateral heat transport processes can be neglected. Consequently, the vertical heat losses through the pillar are less important with respect to the positively-biased device. From Fig. 10, the difference in temperature between these two regimes is more than two orders of magnitude. Also the surface temperature distribution for the negatively-biased device shows a flatter profile. Furthermore, a temperature drop in the center (link) clearly indicates a heat sink because of the pillar and the fact that the heat source is more expanded from the center compared to the positive bias regime. When increasing the applied power beyond $0.1 \mu \mathrm{W}$, some problems related to the convergence of the solutions occurred in the simulator. However, from Fig. 11, it can clearly be seen that in the case of a negative bias, the power required to increase the surface temperature within one degree decreases with increasing total applied power. Extrapolating, one can expect a value of a few nanowatts per Kelvin for a power of $0.2 \mu \mathrm{W}$. On the other hand, for the positively-biased device, the power per Kelvin gradually increases with applied power. The difference between the two curves in Fig. 11 is more than three orders of magnitude.

\section{SENSING PROPERTIES}

It is important to note that during all the experiments on sensing, a constant current mode was used and voltage applied to the device was measured versus time. It has also to be emphasized that the devices are not only intended to maintain certain 


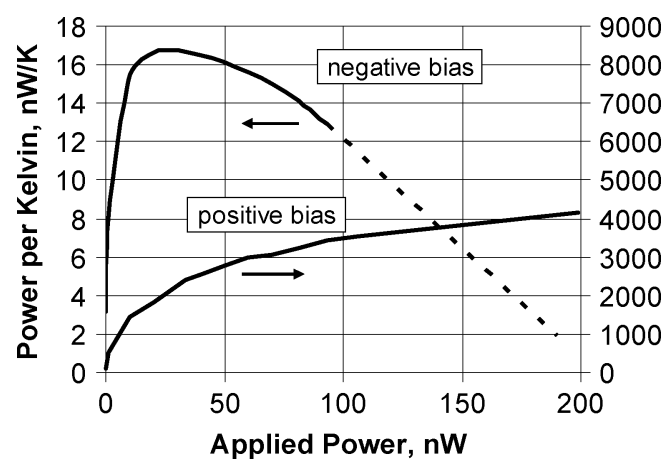

Fig. 11. Power needed per one degree to increase the average surface temperature (simulated surface temperature is averaged for a surface of $1.5 \mu \mathrm{m}$ in diameter) plotted versus total applied power. A comparison between applying a positive bias and applying a negative bias to the poly-Si electrode is shown. For more details see Fig. 10 .

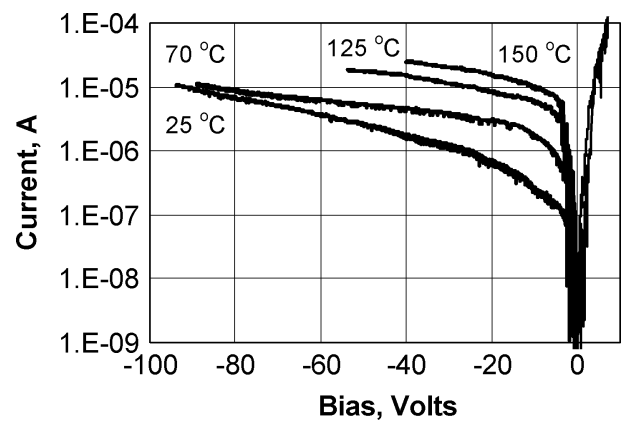

Fig. 12. $I-V$ characteristics of a PSA-device at different substrate temperatures; bias is applied to the poly-Si electrode.

temperature on the surface but also monitor the temperature change, which is a measure of the surface process. As depletion effects in the pillar cause a high device resistance at a negative bias, any energy source capable of generating extra carriers in the depleted pillar will enhance the electrical conductivity. As an example of such energy sources, effects caused by illumination with light and substrate heating are demonstrated in this section. Only experiments at a negative bias are discussed here.

It appears that the link-antifuse can be used as a sensitive thermo-resistor with the sensitivity of about $0.6-0.7 \mathrm{~V}$ per degree centigrade (see Fig. 12). This value was estimated from the following considerations. At room temperature, about $-90 \mathrm{~V}$ should be applied to obtain a current of $10 \mu \mathrm{A}$. At an elevated temperature of $150^{\circ} \mathrm{C}$, applying only $-10 \mathrm{~V}$ was sufficient to obtain the same current. The ratio of the voltage difference to the temperature difference (i.e., $80 \mathrm{~V} / 125^{\circ} \mathrm{C}=0.64 \mathrm{~V} /{ }^{\circ} \mathrm{C}$ ) gave us a rough estimation of the sensitivity. With such high sensitivity, monitoring of exothermic and endothermic processes on the device surface looks feasible. Generation of extra carriers in the pillar during the illumination with light also results in a drop of the resistance (see Fig. 13). As expected, the sensitivity to light decreases with increasing temperature. This is because both the heat and light provide extra carriers and can independently suppress the depletion effects. In Fig. 13, one can notice that the response to light is hard to observe when the temperature exceeds $125^{\circ} \mathrm{C}$.

We carried out a number of experiments on vapor sensing, to clarify whether the PSA-devices are practically able to generate

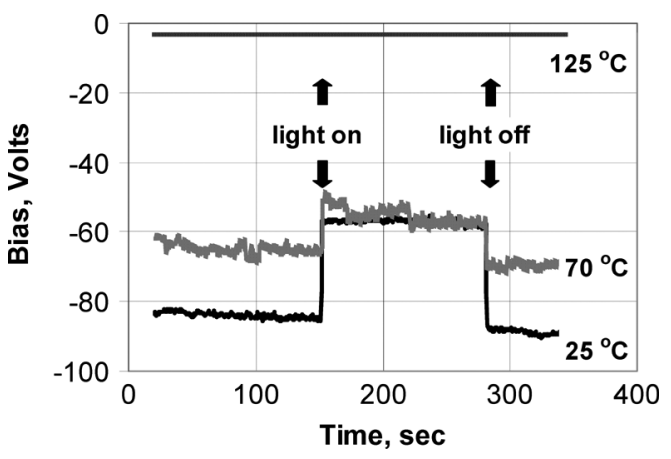

Fig. 13. Sensitivity to external light at different substrate temperatures during a constant current stress of $0.1 \mu \mathrm{A}$.

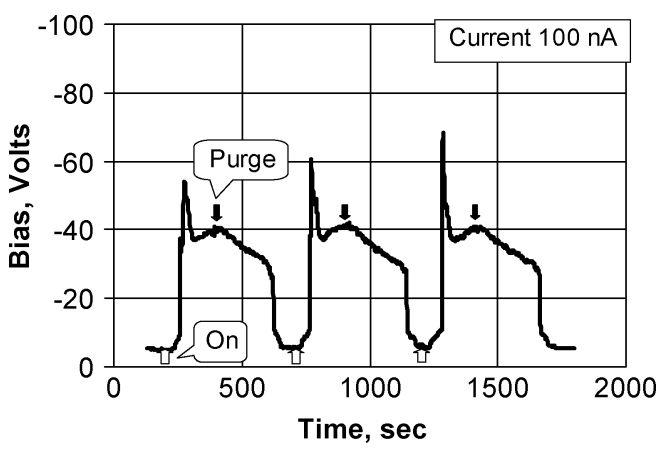

Fig. 14. Device response (i.e., adsorption-desorption cycles in a porous $350-\mathrm{nm}$ thick SOG layer) on $1.3 \%$ ethanol vapor mixed with air. Maximum applied power is $4 \mu \mathrm{W}$, and standby power is $0.5 \mu \mathrm{W}$; on-ethanol flow is turned on, purge-ethanol flow is turned off.

sufficient heat for activation of surface processes and, at the same time, monitor the surface temperature changes caused by such processes. We successfully observed physical adsorption and chemisorption of ethanol, acetone, 2-propanol, and water vapours. If not explicitly mentioned in the text, the PSA-devices were coated with a porous 350 -nm thick SOG layer.

\section{A. Physical Adsorption}

During the experiments on measuring the physical adsorption, the surface temperature was not sufficiently high for chemisorption/decomposition of the precursors. The devices were able to sense the adsorption/desorption cylces in the porous SOG layer, which affected the surface temperature. Namely, after the precursor vapour was introduced into the chamber containing air (see Fig. 14), it could penetrate into the pores and adsorb or condense there. Such an adsorption increased mass of the layer, which led to cooling the surface. This caused the resistance increase. As a consequence, an extra power (i.e., higher voltage) should be applied to maintain the same current. This extra power increased the surface temperature and enhanced the desorption, which caused again cooling the surface and again required applying an extra power to maintain the same current. Such a self-amplifying mechanism resulted in a very sharp increase of the bias after introducing the precursor vapor. Similar effects were observed for water, acetone, ethanol, and 2-propanol. A high response to water vapour pointed out that the temperature was insufficient to 


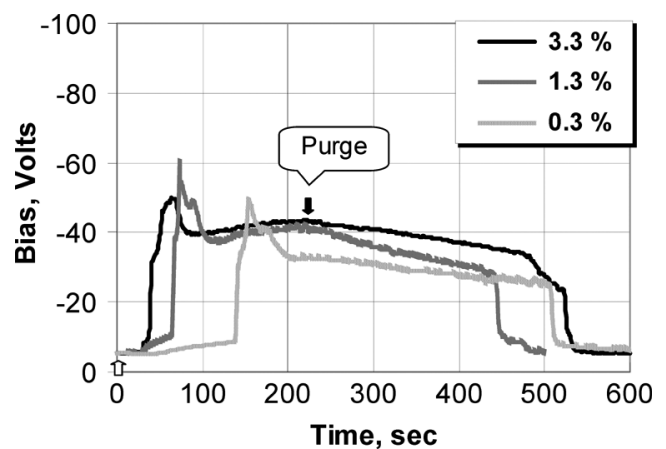

Fig. 15. Response delay for various concentrations of ethanol vapour in air. Ethanol flow is turned on at $0 s$; purge —ethanol flow is turned off.

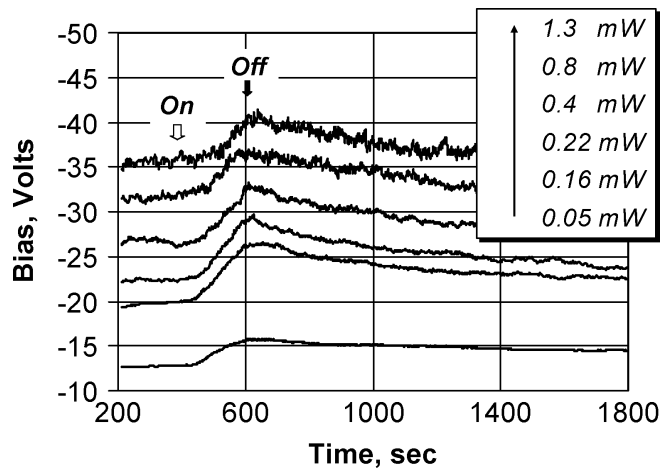

Fig. 16. Device response (i.e., chemisorption-decomposition) after introducing $2 \%$ ethanol vapor in air into the chamber. Applied power is shown in the legend; on - ethanol flow is turned on; off_-ethanol flow is turned off.

suppress the physical adsorption of water. The average dissipated power was still in the $\mu \mathrm{W}$ range. With increasing the standby power up to $10 \mu \mathrm{W}$, we noticed a suppression of the water adsorption, indicating a higher surface temperature. With further increase of power up to hundreds of microwatts, we achieved even higher surface temperatures, likely enabling thermal decomposition of organic compounds, which will be shown in the next subsection.

For the regime of physical adsorption, we additionally observed a response delay depending on vapor concentration (see Fig. 15). As expected, the delay is more pronounced for lower concentrations. This is due to the fact that, for low concentations, the adsorption is limited by the supply. The response delay effect can be utilized, e.g., for a chromathographycal analysis.

\section{B. Chemisorption/Decomposition}

By further increasing the dissipated power, one can expect a suppression of the physical adsorption due to higher surface temperatures. The PSA-devices become insensitive to water vapor at dissipated powers between 10 and $100 \mu \mathrm{W}$. An increase of the power beyond this value leads to a higher surface temperature probably enabling chemisorption of the precursors. Fig. 16 shows the responses to ethanol vapor at different applied powers. Similar results were obtained for acetone and 2-propanol vapor. To check if the thermal conductivity change plays a role, we tested chloroform vapor. At such elevated temperatures, chloroform has 3 times lower thermal conductivity than air [23]. No effect was observed. The peak direction

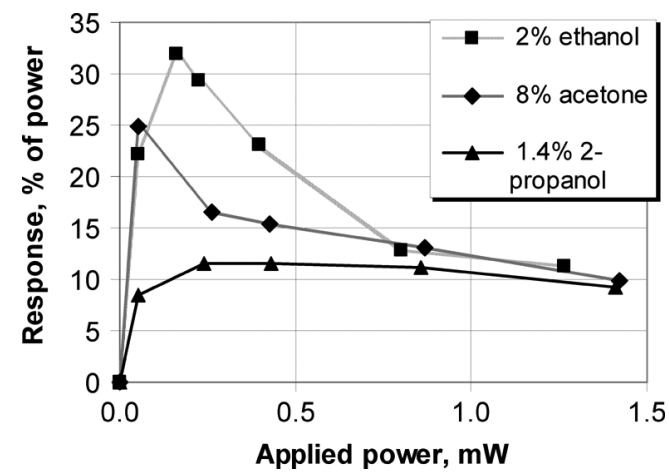

Fig. 17. Response peak (i.e., the peak shown in 0) intensity calculated in percentage points of the applied power and plotted versus applied power for different precursor vapors in air.

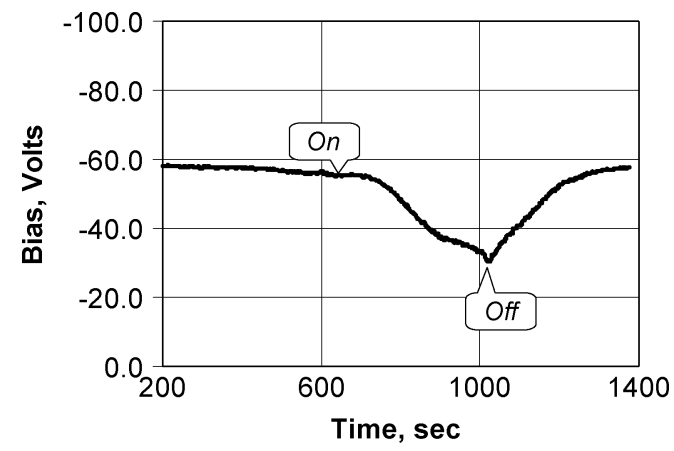

Fig. 18. Response of a PSA-device without a porous 350-nm thick SOG layer on top after introducing a mixture of $18 \%$ of acetone vapor in air; a low power of $0.3 \mu \mathrm{W}$ is applied; on-acetone flow is turned on, and off-acetone flow is turned off. A similar response was observed for $3.5 \%$ of ethanol vapor in air.

upwards in Fig. 16 can be explained by cooling the surface. This can be caused by an endothermic reaction, e.g., thermal decomposition of the precursor vapor occurring inside pores. In this sense, the devices enable monitoring the chemisorption.

\section{Selectivity}

An important result is that all the tested precursor vapors exhibit different responses depending on applied powers. Plotting the response peak intensity (expressed in percents of the applied power) versus applied power, a particular precursor can be identified (see Fig. 17). This rather promising result makes it possible to distinguish between several precursors in a mixture when an array of such sensors operating at various powers is used. The selectivity is clearly present for input powers lower than $0.5 \mathrm{~mW}$ and disappears for powers beyond $1 \mathrm{~mW}$. This can be related to the decay of both the non-radiative heat generation efficiency and sensitivity to temperature with increasing input power, due to a bigger size of the antifuses programmed at higher powers.

\section{Uncoated Devices}

We carried out the sensitivity experiments for several PSAdevices without an SOG layer on top. It was noticed that thermal conductivity did not play a role (i.e., no response after a substitution of synthetic air with $\mathrm{CO}_{2}$ was observed). From the fact that the response peak is directed downwards (see Fig. 18), we can further conclude that the device resistance decreases after the 
vapors are introduced into the measuring chamber. This change of the resistance can be attributed to the temperature increase caused by an exothermic process (e.g., combustion) near the device surface. If such a process takes place, the produced extra heat will increase the device temperature and cause the resistance drop according to Fig. 18. The autoignition temperature is $538^{\circ} \mathrm{C}$ for acetone and $363^{\circ} \mathrm{C}$ for ethanol [23]. This can give a rough indication of the surface temperature. It is important to bear in mind that no catalytic layer aiming at decreasing the combustion temperatures was deposited on the device surface. Therefore, the observed nanopellistor effect occurred at the autoignition temperatures. To enable a highly sensitive Pellistor, the heating element is normally coated with a porous material. This is done to increase the ratio between the combustion power (combustion heat) and the electric power applied to the sensor. If the combustion power is negligible compared to the electric power, it can hardly influence the stand-by device temperature and, therefore, can hardly be detected. To demonstrate the nanopellistor effect for the uncoated PSA-devices, reasonably high concentrations (i.e., $18 \%$ of acetone and $3.5 \%$ of ethanol vapor) were therefore introduced into the chamber.

\section{CONCLUSIONS}

Both the experiments and simulations indicate that the PSA-devices can enable surface temperatures as high as a few hundred degrees centigrade at a power consumption ranging from (sub) $-\mu \mathrm{W}$ to $\mathrm{mW}$. Such a temperature is sufficient for the successful actuation of physical and chemical processes on the surface. The size of the hot area and the temperature can be manipulated by the sign of the applied bias. Two different heat-generation mechanisms (i.e., dissipation at a resistor and a non-radiative recombination of carriers) play a role. In addition, the PSA-devices exhibit a high sensitivity to external heat (or light) and, therefore, can monitor surface temperature changes (or light emission) caused by physical and chemical processes on the surface. These properties together may allow the devices to be used, e.g., in a versatile miniature lab-on-a-chip for sensing and actuating nanoscopic quantities of matter. We demonstrated the device feasibility to perform as a temperature-, adsorption-, and desorption sensor, and as a device to initiate chemisorption/decomposition of the precursors, i.e., a micro-reactor.

The devices were entirely manufactured using silicon-compatible technology steps. A direct connection to an integrated circuit on the same piece of silicon is therefore feasible. The easy manufacturing and system integration is a key benefit of such devices.

\section{REFERENCES}

[1] A. Hulanicki, S. Geab, and F. Ingman, "Chemical sensors: Definitions and classification," Pure Appl. Chem., vol. 63, no. 9, pp. 1247-1250, 1991.

[2] M. Gall, "Si-Planar-Pellistor array, a detection unit for combustible gases," Sensors and Actuators B: Chemical, vol. B16, no. 1-3, pp. 260-264, 1993.

[3] C. Dücsö, M. Ádám, P. Fürjes, M. Hirschfelder, S. Kulinui, and I. Bársony, "Explosion-proof monitoring of Hydrocarbons by Micropellistor," in Proc. Eurosensors-Book of Abstracts, J. Saneistr and P. Ripka, Eds., 2002, pp. 525-526.
[4] V. E. Houtsma, "Gate oxide reliability of poly-Si and poly-SiGe CMOS devices," Ph.D. dissertation, Univ. Twente, Enschede, The Netherlands, 1999.

[5] L. M. Phuong, "Silicon light emitting devices for integrated applications," Ph.D. dissertation, Univ. Twente, Enschede, The Netherlands, 2003.

[6] A. Y. Kovalgin, J. Holleman, and G. Iordache, "A micro-scale hotsurface device based on non-radiative carrier recombination," in Proc. 34th Eur. Solid-State Device Research Conf., R. P. Mertens and C. L. Clayes, Eds., 2004, pp. 353-356, IEEE.

[7] A. Y. Kovalgin, J. Holleman, and A. van den Berg, "A novel approach to low-power hot-surface devices with decoupled electrical and thermal resistances," in Proc. Eurosensors-Book of Abstracts, J. Saneistr and P. Ripka, Eds., 2002, pp. 31-32.

[8] A. Y. Kovalgin, J. Holleman, and G. Iordache, "A versatile microscale silicon sensor/actuator with low power consumption," in Proc. 4th IEEE Conf. Sensors, 2005, pp. 1225-1228.

[9] A. van der Drift, "Evolutionary selection, a principle governing growth orientation in vapour-deposited layers," Philips Res. Repts, vol. 22, p. 267, 1967.

[10] J. Y.W. Seto, "The electrical properies of polycrystalline silicon films," J. Appl. Phys., vol. 46, no. 12, pp. 5247-5254, 1975.

[11] E. Luder, "Polycrystalline silicon-based sensors," Sensors and Actuators, vol. 10, pp. 9-23, 1986.

[12] N. M. Ravindra, S. Abedrabbo, W. Chen, F. M. Tong, A. K. Nanda, and A. C. Speranza, "Temperature-dependent emissivity of silicon-related materials and structures," IEEE Trans. Semiconductor Manuf., vol. 11, no. 1, pp. 30-39, Feb. 1998.

[13] G. E. Jellison, Jr. and D. H. Lowndes, "Optical absorption coefficient of silicon at $1.152 \mu$ at elevated temperatures," Appl. Phys. Lett., vol. 41, no. 7, pp. 594-596, 1982.

[14] G. E. Jellison, Jr. and F. A. Modine, "Optical functions of silicon at elevated temperatures," Appl. Phys. Lett., vol. 76, no. 6, pp. 3758-3761, 1994.

[15] A. T. Fiory and N. M. Ravindra, "Light emission from silicon: Some perspectives and applications," J. Electron. Materials, vol. 32, no. 10, pp. 1043-1051, 2003.

[16] M. Asheghi, K. Kurabayashi, R. Kasnavi, and K. E. Goodson, "Thermal conduction in doped single-crystal silicon films," J. Applied Phys., vol. 91, pp. 5079-5088, 2002.

[17] A. D. McConnell, S. Uma, and K. E. Goodson, "Thermal conductivity of doped polysilicon layers," J. Microelectromech. Syst., vol. 10, pp. 360-369, 2001.

[18] M. Asheghi, Y. K. Leung, S. S. Wong, and K. E. Goodson, "Phononboundary scattering in thin silicon layers," Applied Phys. Lett., vol. 71, pp. 1798-1800, 1997.

[19] T. Yamane, N. Nagai, S.-I. Katayama, and M. Todoki, "Measurement of thermal conductivity of silicon dioxide thin films using a $3 \omega$ method," J. Applied Phys., vol. 91, pp. 9772-9776, 2002.

[20] B. Revaz, B. L. Zink, and F. Hellman, "Si-N membrane-based microcalorimetry: Heat capacity and thermal conductivity of thin films," Thermochimica Acta, vol. 432, pp. 158-168, 2005.

[21] Y. S. Toulokian, P. E. Liley, and S. C. Saxena, "Thermophysical properties of matters," in Thermal Conductivity, Nonmetallic Liquid and Gases. New York: IFI/Plenum, 1970, vol. 3.

[22] A. Melling, S. Noppenbeger, M. Still, and H. Venzke, "Interpolation correlationsfor fluid properties of humid air in the temperature range $100{ }^{\circ} \mathrm{C}$ to $200{ }^{\circ} \mathrm{C}$," J. Phys. Chem. Reference Data, vol. 26, pp. 1111-1123, 1997.

[23] "CRC Handbook of Chemistry and Physics," 73rd ed. D. R. Lide, Ed., 2000, pp. 6-172.

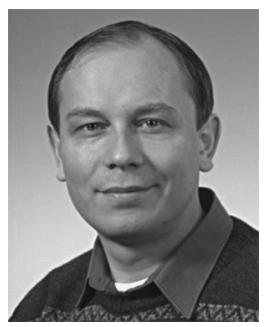

Alexey Y. Kovalgin received the Ph.D. degree in Electronic Materials Technology from the St. Petersburg State Technical University, St. Petersburg, Russia, in 1995.

In 1997, he joined the University of Twente, Enschede, The Netherlands as a postdoctoral researcher. He was involved in CVD, plasma processing, and characterization of thin films. Since 2001, he has been an Assistant Professor at the Chair of Semiconductor Components, University of Twente, where he is involved in thin film deposition technologies, design, and realization and characterization of new silicon devices. He is the author of over 90 reviewed international journal and conference papers. 


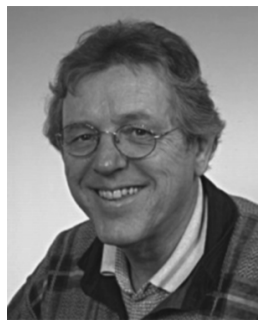

Jisk Holleman received the Ph.D. degree from the University of Twente, Enschede, The Netherlands, in 1993.

His scientific focus was in the past on IC processing and, more recently, on LPCVD, ALD, ICP-CVD and nano-scale diode antifuse devices for light- and heat-based sensors and actuators and LED's in Si. From 1983 to 2003, he was an Assistant Professor with the Department of Electrical Engineering, University of Twente, at the chair of Semiconductor Components. Since 2003, he has been an Associate Professor at the University of Twente. He has contributed to over 100 journal and conference papers in the past 20 years.

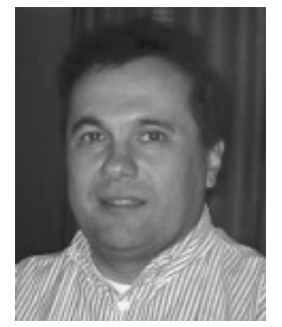

Gheorghe (Gigi) Iordache received the Ph.D. degree in condensed matter physics from the Institute of Atomic Physics, Bucharest, Romania, in 1998.

Since that time, he has been a Research Scientist with the Department of Semiconductor Physics, the National Institute R\&D of Materials Physics, Bucharest, Romania. From 2001 to 2003, he joined the University of Twente, Enschede, The Netherlands, for a one-and-half-year postdoctoral stay. His scientific focus was on thin-film structures for high-power diode laser mirror coatings, design and characterization of diode laser structures, and, more recently, on nano-scale antifuse devices for light- and heat-based sensors and actuators. He is the author of over 30 journal papers and conference contributions and holds three patents. 